\title{
HOW DEMOCRATIC IS THE AMERICAN CONSTITUTION? LA ACTUALIDAD CONSTITUCIONAL EN EE UU EN 2005
}

\author{
ANTONIO DE CABO \\ Profesor de Derecho Constitucional \\ Universidad Complutense \\ MARCOS CRIADO \\ Profesor de Derecho Constitucional \\ Universidad de Alicante
}

SUMARIO

I. Control judicial formal vs. Control judicial sustantivo.

II. Los déficits democráticos de la Constitución de Virginia.

III. Argumentos formales y sustantivos en el caso de las escuchas sin autorización judicial.

IV. Jurisprudencia.

Why should we feel bound today by a document produced more than two centuries ago by a group of fifty-five mortal men, actually signed by only thirty-nine, a fair number of whom were slaveholders, and adopted in only thirteen states by the votes of fewer than two thousand men, all of whom are long since dead and mainly forgotten?

ROBERT DAHL, How Democratic is the American Constitution?

La actualidad constitucional estadounidense, a día de hoy, está marcada por dos hechos que, en parte, están fuera del periodo temporal al que se limi- 
ta esta panorámica: el año 2005. De una parte, la sentencia del Tribunal Supremo en Hamdan v. Rumsfeld, de 29 de junio de 2006, que declara que el Presidente invadió competencias del Congreso al crear los tribunales militares que debían juzgar a los detenidos en Guantánamo. De otra, el descubrimiento del programa de espionaje de llamadas telefónicas y comunicaciones electrónicas de ciudadanos y residentes en EEUU, desarrollado en secreto por la Agencia Nacional de Seguridad, por orden del Presidente, desde 2002.

Respecto a la Sentencia, puede parecer sorprendente que el Tribunal Supremo resuelva el caso de los detenidos en Guantánamo desde la perspectiva de la separación de poderes, y no desde el punto de vista de los derechos humanos en juego y el alcance de las limitaciones que pueden sufrir en tiempos de necesidad que, como vimos en la entrega anterior, era el planteamiento adelantado por la doctrina ${ }^{1}$. Sin embargo, como veremos más adelante, del estudio de las sentencias del Tribunal Supremo en periodos excepcionales, se desprende que este ha sido el criterio más utilizado por la Corte, mientras que el control basado en la ponderación entre necesidad y derechos humanos se aplica a los casos que no están directamente relacionados con las decisiones basadas en poderes excepcionales ${ }^{2}$.

Respecto a las escuchas, el mayor temor de la Administración Bush Jr., la apertura de una investigación por parte de la Comisión de Inteligencia del Senado, ha sido conjurado mediante la concesión de una serie de exigencias de los senadores republicanos, entre ellas una ley de vigilancia antiterrorista que deberá controlar la actividad de la Agencia Nacional de Seguridad. Sin embargo, siguen en curso dos procesos judiciales abiertos por la Unión Americana de Libertades Civiles, y habrá que esperar al fallo de los jueces. En esta panorámica realizaremos un análisis crítico de los argumentos presentados por el Departamento de Justicia en defensa del programa de espionaje.

Gran parte de la producción doctrinal estadounidense del año 2005 continua encontrando fuertes contradicciones entre las políticas de la Administración Bush Jr. y el sistema de derechos constitucionalmente establecido ${ }^{3}$, aunque como veremos, se va imponiendo un marco de análisis según el cual la judicial review de los actos del poder político debe basarse en criterios formales de competencia (institutional process conceptions) y no en la consideración sus-

1 Cfr. Marcos CRIADO y Antonio DE CABO, Whose Voice?: La actualidad constitucional en EEUU en 2004, Teoría y Realidad Constitucional, n'. 16, 2005, pp. 419-431.

2 Un análisis desde el punto de vista competencial de la regularidad de los tribunales militares, puede encontrarse en Shaden YOUSEF, Military Tribunals: Cure for the Terrorism or a Plague All Their Own?, 42 Houston Law Review, 2005, pp.911 ss.

3 Resultan estimulantes Elana J. ZEIDE, In Bed with the Military: First Amendment Implications of Embedded Journalism, 80 New York University Law Review, octubre de 2005, pp. 1309 ss, que analiza desde el punto de la libertad de prensa el programa de introducción de periodistas en las tropas que invadieron Irak; y Matthew J. PEED, Blacklisting as a Foreign Policy: The Politics and Law of Listing Terror, 54 Duke Law Journal, 2005, pp. 1321 ss, donde se analizan las consecuencias legislativas que el Congreso otorga a las listas negras de estados y organizaciones terroristas realizadas discrecionalmente por el Departamento de estado, desde el punto de vista del principio de igualdad. 
tantiva de los derechos fundamentales como límites objetivos del poder (rightsoriented conceptions). De esta suerte, la reciente corriente doctrinal, de la que dábamos noticia en la entrega anterior, partidaria de una participación efectiva de los poderes legislativo y ejecutivo en la interpretación de las disposiciones constitucionales ${ }^{4}$, parece imponerse en la práctica.

\section{CONTROL JUDICIAL FORMAL VS. CONTROL JUDICIAL SUSTANTIVO}

En EE UU el culto la Constitución de Virginia y a los Framers es una suerte de religión civil ${ }^{5}$, y como tal, algo que está fuera tanto de la crítica académica como de la respuesta social. Esta mitificación constitucional tiene importantes consecuencias. En el plano teórico, supone afirmar la continuidad entre los valores, intereses y preferencias de los constituyentes de finales del siglo xviII y la sociedad norteamericana actual. De esta manera, el idealismo norteamericano no consiste en una valoración externa de la Constitución desde una teoría de la democracia o del buen gobierno, sino en la idealización del pasado. Para un cierto sector de la doctrina estadounidense, la desconfianza hacia el poder se materializa sólo en el tiempo presente, cuando la interpretación de la Constitución por el Tribunal Supremo y su aplicación por los poderes legislativo y ejecutivo, ha ido desconociendo el pretendido significado original de las instituciones constitucionales, consistente en una suerte de "originalismo libertario" que afirma el carácter absoluto de las libertades civiles individuales frente a la decisión colectiva ${ }^{6}$. Por ello, no es extraño que gran parte de los estudios históricos de la Constitución de 1787 desarrollados por la doctrina estadounidense no se orienten a relativizar la actuación de los Framers, sino a "descubrir" el sentido original de las regulaciones constitucionales.

4 Vid. Louis FISHER, Constitutional Dialogues: Interpretation as political process, Princeton, Princeton University Press, 1988 ; Larry D. KRAMER, The people themselves : popular constitutionalism and judicial review, Oxford, Oxford University press, 2004; Mark TUSHNET, Taking the Constitution Away From the Courts, Princeton, Princeton University Press, 1999; Neal KUMER KAYTAL, Legislative Constitutional Interpretation, 50 Duke Law Review, 2001, pp. 1355 ss.; Michael STOKES PAULSEN, The Most Dangerous Branch: Executive Power to Say What the Law Is, 83 Georgia Law Review, 1994, pp. 217 ss; Keith E. WHITTINGTON, Extrajudicial Constitutional Interpretation: Three Objections and Responses, 80 North Carolina Law Review, 2002, pp. 773 ss.

5 En una reseña al libro de Robert DAHL, leemos: «... the constitution is beyond criticism. The American civic religion affords it Biblical or Koranic status, even to the point of seeing it as divinely inspired. It's the flag in prose. It's something to be venerated. It's something to be preserved, protected, and defended, as the President swears by God to do... [T]he Constitution of the United States is emphatically not something to be debunked..." Hendrik HERTZBERG, Framed Up: What the Constitution Gets Wrong, New Yorker, 29 de Julio de 2002, p. 85.

6 Una actualización de este "originalismo libertario" puede encontrarse en la obra de Randy E. BANETT, Restoring the Lost Constitution: The Presumption of Liberty, Princeton University Press, Princeton, 2004. Una revisión crítica de estas posiciones puede consultarse en Samuel T. Morison, The Crooked Timber of Liberal Democracy, 2005 Michigan State Law Review, Summer, pp. 461-481. 
Desde el punto de vista práctico, los estudios históricos así orientados resultan funcionales al "originalismo" como cánon interpretativo estático de la Constitución por parte de los jueces ${ }^{7}$, individuado en el conocido como paradigma de la "interpretación contemporánea", según el cual los jueces deben presumir que las primeras leyes dictadas por el Congreso suponen una evidencia fuerte del verdadero significado de la Constitución ${ }^{8}$.

La base de la "interpretación contemporánea", la presunción de la regularidad constitucional de la práctica histórica, unida a ciertos cánones interpretativos derivados de la "dificultad contramayoritaria" propia de la revisión judicial de los actos del poder político, singularmente la presunción de constitucionalidad de tales actos y la interpretación de las leyes ambiguas evitando aquellas lecturas que puedan plantear problemas de constitucionalidad (constitutional avoidance), dan lugar a un paradigma interpretativo - la «interpretación práctica" — que afirma la constitucionalidad de aquellas prácticas gubernativas reiteradas históricamente de manera consistente? . De esta suerte, el control de constitucionalidad de las leyes y actos normativos tiende a centrarse en la regularidad procedimental, a costa de la consideración de los derechos fundamentales como principios sustantivos del ordenamiento jurídico y, por tanto, como límites objetivos de la acción de los poderes públicos.

7 Una aproximación al "originalismo" y a otros cánones de interpretación judicial de la Constitución pueden encontrarse en Marcos CRIADO y Antonio DE CABO, A vueltas con la rama menos peligrosa: La actualidad constitucional en EEUU en 2003, Teoría y Realidad Constitucional, n. 14, 2. ${ }^{\circ}$ semestre 2004, p. 459.

8 Cfr. Myers v. United States, 272 U.S. 52, 175 (1926) ("[A] contemporaneous legislative exposition of the Constitution, when the founders of our government and framers of our Constitution were actively participating in public affairs... fixes the construction to be given its provisions"); Marsh v. Chambers, 463 U.S. 783, 790 (1983); más recientemente, Eldred v. Ashcroft, 537 U.S. 186, 237 (2003) donde el Tribunal establece que la fuerte presunción de constitucionalidad no puede aplicarse a la actividad del Congreso de 1831, puesto que ningún miembro del mismo había sido delegado en la Convención de Virginia. Un análisis de la jurisprudencia del Tribunal Supremo a este respecto se encuentra en Jason T. BURNETTE, Eyes on Their Own Paper: Practical Construction in Constitutional Interpretation, 39 Georgia Law Review, 2005, pp. 1065-1108.

9 Cfr. Ibid., p. 1069. El autor analiza sentencias tanto del Tribunal Supremo como de los tribunales de distrito desde 1803 hasta 2004 para individuar un cánon interpretativo poco conocido y denominado "practical construction", consistente en la búsqueda de un evidencia extrínseca, en concreto una práctica institucional histórica, que por sí misma sirve para probar la validez constitucional de la propia práctica, incluso cuando es ésta la que se pone en cuestión, como se establece en United States v. Midwest Oil Co., 236 U.S. 459, 473 (1915). Así, la práctica institucional reiterada deviene constitucional en aras de un particular principio de seguridad jurídica consistente en el respeto de la estabilidad y las expectativas creadas por una práctica histórica. El profesor Michael Glennon ha analizado estas prácticas históricas en el contexto de la jurisprudencia que resuelve casos relativos a la separación de poderes, estableciendo que los tribunales, para otorgar plena eficacia normativa a la práctica histórica, deben atender a la consistencia, frecuencia, duración, densidad, continuidad y normalidad de la práctica. De todas ellas, las decisiones judiciales sugieren que la consistencia de la práctica es el mínimo requerido y por tanto debe estar siempre presente. Además, para tener efectos legales, la práctica debe tener la cualidad de opinio juris, en el sentido de que la práctica debe ser percibida como una norma jurídica. Cfr. Michael J. GLENNON, The Use of Custom in Resolving Separation of Powers Disputes, 64 Berkley University Law Review, 1984, pp. 129-134. 
Un ejemplo de esta labor interpretativa basada en el procedimiento y no en los derechos, nos la brinda el análisis de la jurisprudencia del máximo tribunal desde 1941 hasta nuestros días, realizada por Lee Epstein, Daniel E. Ho, Gary King y Jeffrey A. Segal. En la entrega anterior veíamos cómo la jurisprudencia y la doctrina norteamericana se dividían respecto al papel que debe jugar el Tribunal Supremo en tiempos de emergencia y peligro ${ }^{10}$, entre quienes consideran que los jueces deben desarrollar un papel especialmente vigilante en la protección de los derechos y libertades en épocas de conmoción ${ }^{11}$, y la posición mayoritaria de quienes consideran que silent enim leges inter arma y que los jueces deben estar especialmente dispuestos a subordinar derechos y libertades cuando el país está amenazado ${ }^{12}$. La idea de que el Tribunal actúa limitando o suspendiendo derechos y libertades en condiciones de amenaza está ampliamente aceptada en los EEUU post-11 de septiembre, como lo ha estado desde la Primera Guerra Mundial ${ }^{13}$, lo que ha llevado a la doctrina a estimar que, vigente un periodo de excepción, el Tribunal Supremo actúa limitando los derechos fundamentales según las exigencias de la seguridad nacional.

10 Cfr. en general, Marcos CRIADO y Antonio DE CABO, Whose Voice? La Actualidad Constitucinal en EEUU en 2004, cit., pp. 419-425.

11 Lo que se correspondería con lo establecido por el Tribunal Supremo en la sentencia Ex parte Milligan, 71 U.S. (4 Wall.) 2, 120-121, 123-124 ("The Constitution of the United States is a law for rulers and people, equally in war and in peace, and covers with the shield of its protection all classes of men, at all times, and under all circumstances... but if society is disturbed by civil commotion... these safeguards need, and should receive, the watchful care of those instructed with the guardianship of the Constitution and the Laws"). Cfr. Oren GROS, Chaos and Rules: Should Responses to Violent Crises Always Be Constitutional?, 112 Yale Law Journal, 2003, pp. 1043-1044 y Jules LOBEL, The War on Terrorism and Civil Liberties, 63 University of Pittsburgh Law Review, 2002, pp. 769-772.

12 En línea con la sentencia Korematsu vs. United States, 323 U.S. 214, 219-220 (1944) ("All citizens alike, both in and out of uniform, feel the impact of war in grater or lesser measure. Citizenship has its responsibilities as well its privileges, and in time of war the burden is always heavier. Compulsory exclusion of large groups of citizens from their homes, except under circumstances of direst emergency and peril, is inconsistent with our basic governmental institutions. But when under conditions of modern warfare our shores are threatened by hostile forces, the power to protect must be commensurate with the threatened danger"), Cfr. Shira A. SCHEINDLIN, Matthew L. SCHWARTZ, With All Due Deference. Judicial Responsibility in a Time of Crisis, 32 Hofstra Law Review, 2004, pp. 795 ss; Michal R. BELKNAP, A Putrid Pedigree: The Bush Administration's Military Tribunals in Historical Perspective, 38 California Western Law Review, 2002, pp. 433 ss y Donald A. DOWNS, Erik KIUNNEN, A Response to Anthony Lewis: Civil Liberties in a New Kind of War, 2003 Wisconsin Law Review, pp. 385 ss. La cita de Cicerón está tomada de la Pro T. Annio Milone Oratio en su edición italiana Orazione pro Milone, Signorelli, Milán, 1982, p.76.

13 Vid, por ejemplo, Floyd Abrams, The First Amendment and the War Against Terrorism, 5 University of Pennsylvania Journal on Constitutional Law, 2002, pp. 10-11; Philip B. Heymann, Civil Liberties and Human Rights in the Aftermath of September 11, 25 Harvar Journal on Law and Public Policy, 2002, pp. 441 ss; Thomas W. Joo, Presumed Disloyal: Executive Power, Judicial Deference, and the Construction of Race Before and After September 11, 34 Columbia Human Rights Law Review, 2002, pp. 1 ss; Neal K. Kaytal y Laurence H. Tribe, Waging War, Deciding Guilt: Trying the Military Tribunals, 111 Yale Law Journal, 2002, pp. 1259-1260; Anthony Lewis, Civil Liberties in a Time of Terror, 2003 Wisconsin Law Review, pp. 264-266; Mark Tushnet, Defending Korematsu? Reflections on Civil Liberties in Wartime, 2003 Wisconsin Law review, pp. 284-287. 
Sin embargo, el análisis jurisprudencial revela que, en tiempos de guerra y respecto a los casos directamente relacionados con ella, el Tribunal no aborda la cuestión desde una dogmática de los derechos fundamentales, determinando tanto la idoneidad de la seguridad nacional para limitar derechos como el alcance de las limitaciones, sino que se limita a controlar la regularidad procedimental de la norma restrictiva desde una perspectiva institucional ${ }^{14}$. En los casos directamente relacionados con la guerra, el Tribunal Supremo no utiliza la seguridad nacional como un parámetro de control. Sin embargo, y paradójicamente, la Corte utiliza la seguridad nacional como justificación para limitar derechos fundamentales en casos no directamente relacionados con la guerra ${ }^{15}$. Así, pudiendo funcionar la solución a estos casos como precedente, los efectos de la guerra respecto a los casos que involucran derechos fundamentales y que no están directamente relacionados con ella, tienen efectos más persistentes que los casos resueltos desde una perspectiva institucional.

\section{LOS DÉFICITS DEMOCRÁTICOS DE LA CONSTITUCIÓN DE VIRGINIA}

En este contexto, resulta llamativo un libro como el del profesor Robert Dahl "How Democratic is the American Constitution?", cuyo objetivo declarado es provocar un debate público sobre las carencias de la Constitución de 1787 a partir de la crítica del texto constitucional vigente y de la labor de los Framers, que desemboque en una reforma democrática que dé protagonismo al ciudadano en la toma de decisiones y en el control de los poderes constituidos ${ }^{16}$. El libro, resultado de las Castle Lectures impartidas por Robert Dahl en la Universidad de Yale en 2000, se publicó inicialmente en 2001, fue reeditado en 2003 con un nuevo capítulo y en 2005 ha sido analizado por diversos especialistas en un Symposium organizado por la Escuela de Leyes Benjamin N. Cardozo ${ }^{17}$.

El libro renuncia al originalismo como método de abordar el análisis del texto constitucional, por cuanto implica una aceptación acrítica del mismo y por tanto un apriorismo valorativo. El autor plantea tres frentes de crítica. En primer lugar, en cuanto a los orígenes de la Constitución y, en concreto, a la labor de los Framers, el autor considera que acudieron a la Convención de Virginia sin un conocimiento exhaustivo de las instituciones de gobierno de una república y sin posibilidad de contrastar sus convicciones con experiencia constitucional alguna, por lo que actuaron movidos únicamente por sus propios intereses ${ }^{18}$.

14 Cfr. Lee EPSTEIN, Daniel E. Ho, Gary KING y Jeffrey A. SEGAL, The Supreme Court During Crisis: How War Affects Only Non War Cases, 80 New York University Law Review, n.1, abril 2005, pp. 9, 49-58 y passim.

15 Ibid., pp. 9, 88 ss.

16 Cfr. Robert A. DAHL, Comments, 26 Cardozo Law Review, n. 6, 2005, pp. 2541-2548; Id, How democratic is the American Constitution?, New Haven, Yale University Press, 2001, pp. 119 y 154.

17 Vid. el número de mayo de 2005 de la 26 Cardozo Law Review, n.6, 2005, pp. 2513-2551.

18 Robert A. DAHL, How Democratic..., cit., pp. 116-119, 186, 190-191. 
En segundo lugar, en cuanto a los resultados de la aplicación del Texto constitucional comparados con los de los veintiún estados que han tenido sistemas democráticos estables durante más de medio siglo, la Constitución estadounidense ha permitido uno de los niveles más altos de crecimiento económico del mundo, pero también de desigualdad económica y el porcentaje mayor de personas encarceladas; EEUU figura entre los peores países en cuanto a representación femenina en la legislatura, participación en las elecciones, eficiencia en el uso de la energía, gastos en servicios sociales y ayuda exterior ${ }^{19}$.

En tercer lugar, plantea una crítica a la Constitución desde una concepción ideal de la democracia El estado democrático ideal debería proporcionar "derechos, libertades y oportunidades para una participación efectiva... la posibilidad de adquirir una comprensión suficiente de las políticas públicas y sus consecuencias... y medios a través de los cuales los ciudadanos pudieran mantener un control adecuado sobre la agenda de las políticas y decisiones gubernamentales ${ }^{20}$. Es aquí donde Robert Dahl encuentra serios déficits democráticos en la Constitución estadounidense, tanto en la Constitución escrita, como en aquellas prácticas arraigadas que, aún no siendo parte de la Constitución, se consideran como tales, particularmente en el sistema electoral mayoritario, que reduce el pluralismo social a bipartidismo ${ }^{21}$.

Respecto a la elección entre republicanismo y democracia, Dahl se muestra firme partidario de la democracia ${ }^{22}$, entendiendo "republicanismo" como aquel sistema de gobierno en el que sólo unos pocos electos definen qué es el bien común ${ }^{23}$, y por "democracia" aquel sistema en que todos los ciudadanos concurren en la definición del bien colectivo. La Decimoséptima Enmienda otorga a cada estado dos senadores independientemente de la población, lo que resulta una clara violación del principio "un hombre, un voto" ${ }^{24}$. Puesto que estos dos senadores otorgan a los estados dos electores en el colegio que debe elegir al Presidente, la falta de proporción entre voto y población que existe en el Senado, se traslada a la elección presidencial, de suerte tal que los estados

19 Ibid., pp. 117, 168-169.

$20 \mathrm{Ibid}$., p. 136. En la contrarréplica a las intervenciones en el Symposium de la Escuela Cardozo, Robert Dahl hace mención expresa a la práctica de los "deliberative polls" teorizados por James S. FISHKIN, Democracy and Deliberation: New Directions for Democratic Reform, New Haven, Yale University Press, 1991. Cfr. Robert A. Dahl, Comments, cit., p. 2546.

21 Robert A. DAHL, How democratic... cit., pp. 58-59.

22 Una crítica desde el punto de vista republicano a la posición de Robert DAHL puede encontrarse en Marci A. HAMILTON, Republican Democracy is not Democracy, 26 Cardozo Law Review, n. 6, 2005, pp. 2529-2534.

23 Un análisis sobre los orígenes calvinistas del sistema de gobierno republicano puede consultarse en Marci A. HAMILTON, Direct Democracy and the Protestant Ethic, 13 Journal of Contemporany Legal Issues, 2004, pp. 411 ss.

24 Robert A. DAHL, How democratic... cit., pp. 13-15, 47-50, 144-145. Los dos millones de ciudadanos que viven en Nevada eligen a dos Senadores, así como los 34 millones que viven en California. Esto quiere decir que el voto de una persona en Nevada vale casi 17 veces lo que vale el voto de una persona en California. Del mismo modo, el voto de un ciudadano de Wyoming, cuya población no alcanza el medio millón, vale casi 69 veces lo que vale el voto californiano. 
menos poblados tienen un peso mayor en la elección que los más populosos, y puede darse el caso de que quien obtiene más votos en total, pierda las elecciones $^{25}$. El sistema produce un presidente, pero dada la naturaleza de la elección y la naturaleza del cargo, no produce un mandato ${ }^{26}$.

Un tema no central pero sí recurrente del libro es la llamada "dificultad contramayoritaria" que suscita la judicial review ${ }^{27}$. El profesor Dahl no parece preocupado por la revocación de leyes estatales por los tribunales federales ${ }^{28}$, pero sí se muestra crítico con la inaplicación de leyes federales. De un lado, Dahl parece seguir a Ely cuando afirma que el papel esencial de los tribunales, como protectores de los derechos individuales y de las minorías, es el de garantizar un participación completa e igual en el proceso político ${ }^{29}$. De otro lado, el autor apunta hacia una interpretación objetiva de los derechos fundamentales cuando nos dice que a la hora de pensar los derechos hay que tener en cuenta los deberes correlativos, la oportunidad de ejercicio y, sobre todo, los recursos que permiten el ejercicio efectivo de los derechos, singularmente los recursos que permiten la libertad de expresión y la participación en una elección libre y justa ${ }^{30}$. En este sentido, parece como si la consideración subjetiva de los derechos necesitara de un poder judicial que los hiciera cada vez actuales y efectivos, mientras que la consideración objetiva de los derechos, implicando decisiones sobre la gestión de los recursos escasos, fuera algo reservado a la decisión colectiva, al proceso político, correspondiendo a los jueces garantizar el carácter pluralista y democrático de dicha decisión.

Respecto a la cuestión que planteábamos en la primera parte de la panorámica, Robert Dahl probablemente afirmaría que la legitimidad de las acciones del poder político depende de la efectiva participación de todos los ciudadanos en la decisión, lo que debería corresponderse con el proceso de toma de decisiones legalmente establecido. Sin embargo, en ausencia de un sistema de participación ciudadana como el que idealmente diseña el profesor Dahl, sólo queda en pié el criterio formal de competencia y regularidad en la toma de decisiones, con lo que el argumento, indirectamente, refuerza la práctica jurisprudencial tal y como se ha venido produciendo. Al reducir la legitimidad de las decisiones a la pura participación democrática y hacerla coincidir idealmente con el deber ser de la legalidad, el profesor Dahl no puede extraer de la cons-

25 Ibid., pp. 81-82 y passim. Ello ocurrió en 2000 (según el recuento final, Bush Jr. obtuvo 50.456.002 votos frente a 50.999.897 que obtuvo Gore), en 1824, 1876 y 1888. Cfr. Comisión Federal de Elecciones (http.www.fec.gov).

26 Robert DAHL ya había desarrollado esta idea en The Myth of the Presidential Mandate, 105 Political Science Quaterly, 1990, pp. 355 ss.

27 Robert A. DAHL, How Democratic... cit., pp. 18-19, 54-55, 152-154, 167. Sobre la «dificultad contramayoritaria" Vid. en general Marcos CRIADO y Antonio DE CABO, La actualidad constitucional en Estados Unidos en 1998, Teoría y Realidad Constitucional, n.3, 1999, pp. 297-298.

28 Robert A. DAHL, How Democratic... cit., p. 54.

29 Ibid., pp. 211. Vid. John HART ELY, Democracy and Distrust: A Theory on Judicial Review, Cambridge, Mass., Harvard University Press, 1980.

30 Robert A. DAHL, Comments, cit., p. 2543. 
trucción objetiva de los derechos fundamentales a la que apunta, un criterio objetivo con el que juzgar el contenido de la decisión del poder político, que quedaría así libre de ataduras materiales.

\section{ARGUMENTOS FORMALES Y SUSTANTIVOS EN EL CASO DE LAS ESCUCHAS SIN AUTORIZACIÓN JUDICIAL}

Una aplicación práctica de los argumentos que venimos considerando puede encontrarse en la defensa jurídica de las escuchas telefónicas de ciudadanos estadounidenses ordenadas por el Presidente Bush. El 16 de diciembre de 2005, el New York Times publicó una noticia según la cual el Presidente ordenó en 2002 a la Agencia de Seguridad Nacional (NSA), la intervención y escucha de las llamadas internacionales (y en algunos casos nacionales) y de los e-mails de cientos de miles de ciudadanos y de residentes legales en los EEUU sin autorización ni control judicial, como exigen la Cuarta Enmienda y la Ley de Vigilancia de Inteligencia Extranjera (FISA) ${ }^{31}$.

En 1978, después de de que se destapara un amplio programa de violaciones de la intimidad asociadas a los programas de vigilancia de inteligencia extranjera, el Congreso y el Presidente aprobaron y promulgaron la FISA como la norma general que regula la vigilancia electrónica dentro de los Estados Unidos. Con relativamente pocas excepciones, la FISA autoriza la vigilancia electrónica únicamente bajo ciertas condiciones y sólo mediante la aprobación de un Tribunal especial creado por la propia ley. Únicamente admite escuchas no autorizadas en tiempo de guerra y sólo durante los primeros quince días consecutivos a la declaración de la misma o a la autorización para el uso de la fuerza ${ }^{32}$. Cualquier otra escucha no permitida por la FISA, se considera delito federal ${ }^{33}$, al tiempo que la propia FISA y ciertas normas específicas del Código Criminal Federal (relativas a las escuchas realizadas durante una investigación criminal) establecen slos medios exclusivos a través de los que la vigilancia electrónica.... deberá llevarse a cabo" en los EEUU ${ }^{34}$.

El Presidente admitió su responsabilidad en la autorización de las escuchas, aunque defendió su autoridad para hacerlo. Los argumentos, que inciden en la línea de regularidad institucional y procedimental y no en la idea de que la seguridad nacional sirve como parámetro de limitación de libertades, podemos encontrarlos en la Carta que el Departamento de Justicia remitió el 22 de diciembre de 2005 a los portavoces de la mayoría y de la minoría en las Comisiones de Inteligencia de Congreso y Senado:

En primer lugar, el Departamento de Justicia admite que el tipo de escuchas realizadas por la NSA entran dentro de las cubiertas por la regulación de la FISA y

31 Pub. L. No. 95-511, 92 Stat. 1783.

3250 U.S.C. $₫ 1811$.

33 Id. $\$ 1809$.

3418 U.S.C. $₫ 2511$ (2)(f) (el subrayado es mio). 
que no cumplieron los requisitos exigidos por el texto legal, pero que estaban implícitamente incluidas en la Autorización para el Uso de la Fuerza Militar (AUMF) ${ }^{35}$ otorgada por el Congreso el 18 de septiembre de 2001, que permite al Presidente usar "toda la fuerza necesaria y apropiada para luchar contra Al Qaeda". De la misma manera que en Hamdi $v$. Rumsfeld la mayoría relativa del Tribunal Supremo admitió que la detención militar de combatientes enemigos capturados en el campo de batalla es un "un incidente esencial de la conducción de la guerra" y que por tanto debía considerarse incluida en la Autorización del Congreso, la búsqueda de "señales de inteligencia enemiga", aun cuando conlleve la intervención de teléfonos estadounidenses sin autorización judicial y sin el cumplimiento de requisitos específicos, debe considerarse un "incidente esencial de guerra".

Frente a ello pueden presentarse cuatro contrargumentos:

a) El Departamento de Justicia pretende que la norma general contenida en la AUMF prevalezca sobre la regulación expresa y específica de la FISA, cuando el principio general en caso de conflicto entre disposiciones legales es que la norma específica prevalece sobre la general ${ }^{36}$. El Congreso ha previsto específicamente en caso de guerra que régimen legal tienen las escuchas no autorizadas en el $₫ 1811$ de la FISA, y lo ha limitado a quince días, mientras que el programa de la NSA ha venido desarrollándose durante cuatro años.

b) El argumento del Departamento de Justicia obliga a concluir que el Congreso ha revocado implícitamente la norma que declara que la FISA y ciertas normas del Código Criminal Federal constituyen los medios específicos a través de los que se permite la vigilancia electrónica en EEUU (18 U.S.C. \ 2511 (2)(f)). Sin embargo, la "revocación implícita" cuenta con el rechazo general del Tribunal Supremo, y sólo puede admitirse si se aporta un "evidencia abrumadora "37 de que esa fue la voluntad del Congreso y si las leyes implicadas son irreconciliables ${ }^{38}$. No parece que la AUMF y la sección 2511 (2)(f) sean irreconciliables y no existe evidencia alguna de la intención del Congreso de revocar la norma.

c) El Fiscal General Alberto Gonzales ha admitido que la Administración Bush Jr. No intentó reformar la FISA para autorizar el programa de escuchas de la NSA porque sabía que el Congreso rechazaría la reforma ${ }^{39}$.

35 Pub. L. No. 107-40, 115 Stat. 224 (2001)

36 Cfr., por ejemplo, International Paper Co. v. Oulette, 479 U.S. 481, 494 (1987) y Morales $v$. TWA, Inc., 504 U.S. 374, 384-385 (1992).

37 Cfr. J.E.M. Ag. Supply, Inc. v. Pioneer Hi-Bred Int'l, Inc., 534 U.S. 124, 137 (2001).

38 Id., 141-142, que hace referencia a Morton v. Mancari, 417 U.S. 535, 550 (1974).

39 "We have discussions with Congress in the past - certain members of the Congress - as to wether or not FISA could be amended to allow us to adequately deal with this kind of threat, and we were advised that that would be difficult, if not impossible". Rueda de prensa del Fiscal General Alberto Gonzales y el General Michael HAYDEN, Subdirector para la Inteligencia Nacional, de 19 de diciembre de 2005, en http://www.whitehouse.gov/news/releases/2005/12/20051219-1.html. 
El ejecutivo no puede argumentar al mismo tiempo que el Congreso autorizó el programa en la AUMF y que no le preguntó porque se temía que rechazaría ese programa, máxime cuando en la USA PATRIOT ACT de $2001^{40}$ el ejecutivo consiguió que el Congreso reformara la FISA en distintos aspectos ${ }^{41}$.

d) El respaldo de Hamdi vs. Rumsfeld para afirmar la existencia de poderes implícitos en la AUMF no parece defendible, toda vez que el propio Tribunal Supremo limitó el alcance del incidente esencial de conducción de la guerra" a individuos que "fuesen parte de o sirvieran de apoyo a fuerzas hostiles a los Estados Unidos o sus compañeros de coalición en Afganistán y que estuvieran involucrados en un conflicto armado contra los Estados Unidos, ${ }^{42}$.

En segundo lugar, el Departamento de Estado argumenta que la FISA y la AUMF deben ser interpretadas de manera tal que permitan el programa de vigilancia, porque de otro modo estaríamos ante un conflicto entre la FISA y los poderes que el art. II de la Constitución reconoce al Presidente como Comandante en Jefe. Por tanto, en aplicación del cánon de constitutional avoidance, debe concederse deferencia a la interpretación que de sus propios poderes ha hecho el Presidente. Frente a ello cabe contrargumentar:

a) Los limites establecidos por la FISA son compatibles con los poderes establecidos en el art. II. No cabe duda de que, en ausencia de regulación por parte del Congreso, el Presidente goza de una "autoridad constitucional inherente" para recavar "señales de inteligencia extranjera" sin más límites que los establecidos en la Cuarta Enmienda, y así ha ocurrido en los Estados Unidos hasta $1978^{43}$. Pero la FISA revocó expresamente esa situación al establecer que ella y el Código Criminal eran los medios exclusivos a través de los que se podía espiar a los habitantes de los EEUU. Al hacerlo, el Parlamento no desconoció un poder Presidencial, puesto que no prohíbe la obtención de inteligencia a través del espionaje, sino que lo sometió a un procedimiento razonable de ejercicio para proteger derechos legítimos de los ciudanos, competencia que le reconoce el art. I de la Constitución ${ }^{44}$. Además, el art. II de la Constitución impone

40 Pu. L. No. 107-56, 115 Stat. 272 (2001).

41 Particularmente las secciones 218 (relativa a la extensión de las escuchas admitidas por la FISA a las investigaciones criminales) y la 215 (conocida popularmente como la "libraries provision"). 42542 U.S. 507, 519 (2004).

43 El derecho federal relativo a las escuchas telefónicas preveía expresamente que "nothing contained in this chapter or in section 605 of the Communications Act of 1934 shall limit the constitutional power of the President... to obtain foreign intelligence information deemed essential to the security of the United States". 18 U.S.C. \ 2511 (3) (1976).

44 Así lo estableció el propio Congreso, concluyendo que «even if the President has the inherent authority in the absence of legislation to authorize warrantless electronic surveillance for foreign intelligence purposes, Congress has the power to regulate the conduct of such surveillance by legislating a reasonable procedure, which then becomes the exclusive means by which such surveillance may be conducted" H.R. Rep. No. 95-1283, pt. 1, p. 24 (1978) 
al Presidente la obligación general de cumplir y hacer cumplir las leyes válidamente aprobadas por el Congreso, incluida la FISA ${ }^{45}$.

Durante la Guerra de Corea, el Presidente Truman ordenó la intervención de las fundiciones ${ }^{46}$, paralizadas por una huelga nacional de los trabajadores del metal, amparándose en poderes constitucionales inherentes del poder ejecutivo, particularmente los del Presidente como Comandante en Jefe, y alegando que la huelga ponía en peligro la defensa nacional. El Tribunal Supremo declaró inconstitucional la intervención en la sentencia Youngstown Sheet \& Tube Co. v. Sawyer, asegurando que ello constituía un acto legislativo competencia "exclusiva del Congreso tanto en los buenos como en los malos tiempos ${ }^{47}$. En la sentencia, el juez Jackson diseñó un esquema tripartito de control de los actos del Presidente condicionado a la acción del Congreso. Según este esquema, cuando el Presidente actúa de conformidad con una autorización expresa o implícita del Congreso, goza de "la más amplia latitud de la interpretación judicial"; cuando lo hace en ausencia tanto de otorgamiento como de denegación de autoridad, el control debe hacerse teniendo en cuenta las exigencias de los acontecimientos y los imponderables del momento en que se tomó la decisión, y por tanto sería de aplicación la ponderación entre derechos y seguridad que ha venido diseñando la doctrina estadounidense; por último, cuando el Presidente toma medidas incompatibles con el deseo explícito o implícito del Congreso, el poder presidencial está en su punto más bajo, y el control judicial debe ser más férreo ${ }^{48}$. De esta suerte, los poderes presidenciales no son algo fijo, sino fluctuante, según el ejercicio que de los suyos propios haga el Congreso.

b) La interpretación de la AUMF en el sentido de permitir el espionaje sin autorización judicial, plantea dudas acerca de su constitucionalidad. El canon interpretativo de la constitutional avoidance invocado por el Departamento de Justicia, y consistente en que las leyes ambiguas deben interpretarse de tal manera que no se planteen cuestiones constitucionales serias, puede volverse contra los deseos de la Administración. En primer lugar, el canon de la constitutional avoidance se aplica únicamente en el caso de que la posible inconstitucionalidad sea de importancia, y "no para eliminar todas las posibles disputas acerca de la constitucionalidad de la ley ${ }^{49}$. En ausencia de una cuestión seria sobre la constitucionalidad de la FISA, no hay razón para siquiera plantearse una interpretación de la AUMF en el sentido de invalidar el régimen de la FISA $^{50}$.

45 "... he shall take Care that the Laws be faithfully executed".

46 Exec. Order No. 10.233, 3C.F.R. 425 (1951), que autoriza al Secretario de Comercio a tomar e intervenir las fundiciones y acererías.

47 Youngstown Sheet \& Tube Co. v. Sawyer, cit., 587-89.

48 Id., 635-638.

49 Reno v. Flores, 507 U.S. 292, 314 n. 9 (1993).

50 En 2002, el Tribunal especial creado por la FISA (FISA Court of Review) planteó in dictum que el Congreso no podía invadir el poder constitucional del Presidente de dirigir la vigilancia de la inteligencia extranjera. Cfr. In Re Sealed Case No. O2-001, 310 F.3d 717, 742 (FISA Ct. Rev. 2002). El Tribunal no planteó que la FISA fuera inconstitucional, ni ningún otro tribunal ha sugerido que lo fuera. El fallo se basó en United States v. Truong Dibn Hung, 629 F.2d 908 (4th Cir. 1980), pero en 
En segundo lugar, el Tribunal Supremo nunca ha admitido un poder tan amplio como para invadir la intimidad de los estadounidenses sin la existencia de una sospecha razonable o de una supervisión judicial. El programa de espionaje de la NSA se ha dearrollado sin ninguna de las garantías presumiblemente exigidas por la Cuarta Enmienda para una vigilancia electrónica: causa probable individualizada y autorización escrita o algún otro tipo de orden de un juez o magistrado. El Tribunal Supremo ha establecido desde hace tiempo la necesidad de autorización y causa probable para el caso de escuchas telefóni$\mathrm{cas}^{51}$, y en el único caso en que ha considerado intervenciones telefónicas relacionadas con la seguridad nacional, declaró que la Cuarta Enmienda prohibe las escuchas sin aquellas garantías ${ }^{52}$. Aunque el Tribunal en este caso dejó abierta la cuestión de si la Cuarta Enmienda permite escuchas sin autorización en casos de inteligencia extranjera, como precedente plantea serias cuestiones acerca de la inconstitucionalidad de un poder tan extenso como el que el presidente ejerce al autorizar el programa de escuchas de la NSA ${ }^{53}$.

Basándose en In re Sealed Case No. O2-OO1 ${ }^{54}$, el Departamento de Justicia sostiene que el programa de espionaje de la NSA está dentro de una excepción a las exigencias de autorización y causa probable, consistente en búsquedas razonables que sirven a "necesidades especiales" que van más allá del cumplimiento ordinario de la ley. Sin embargo las "necesidades especiales" no han servido para permitir escuchas sin autorización. Generalmente, se han utilizado para excusar los requisitos de autorización y sospecha individualizada en aquellos casos en que tales requisitos resultaban impracticables y la intrusión en la intimidad es mínima ${ }^{55}$. Pero practicar escuchas telefónicas no es una intromisión mínima en la intimidad, y la experiencia de aplicación de la FISA hasta nuestros días demuestra que es posible vigilar la inteligencia extranjera a través de autorizaciones judiciales basadas en sospechas individualizadas.

\section{JURISPRUDENCIA}

Durante el período cubierto por esta panorámica, el Tribunal Supremo ha dictado 79 sentencias (full opinions), una menos que en el año anterior. La muerte el 3 de septiembre del Magistrado William H. Renquist, Presidente, ha

esta sentencia el tribunal de instancia declaró expresamente la constitucionalidad de la FISA: «the imposition of a warrant requirement, beyond the constituctional minimun described in this opinion, should be left to the intricate balancing performed in the course of the legislative process by Congress and the President" (629 F.2d, 915 n.4).

51 Katz v. United States, 389 U.S. 347 (1967)

52 United States v. United States Dist. Court, 407 U.S. 297 (1972).

53 Id., 316-318 donde explica la dificultad de garantizar las libertades recogidas en la Cuarta Enmienda si el espionaje doméstico se realiza únicamente bajo la discrecionalidad del poder ejecutivo.

54 Vid. supra nota 48.

55 Cfr., por ejemplo, Griffin v. Wisconsin, 483 U.S. 868, 873 (1987). 
sido suplida por el nombramiento del Magistrado John Roberts como nuevo Presidente lo que inaugura, por tanto, según la costumbre norteamericana, una nueva "era" jurisprudencial, cuyos resultados habrán de constatarse en futuras Panorámicas.

\section{Derecho internacional}

En Jose Ernesto Medellin, Petitioner v. Doug Dretke, Director, Texas Department Of Criminal Justice, Correctional Institutions Division [125 S. Ct. 2088 (2005)], se plantearon al Tribunal importantes cuestiones relativas al estatus del derecho internacional en relación con la vinculatoriedad de la decisión Case Concerning Avena and Other Mexican Nationals [(Mex. v. U.S.) (Mar. 31, 2004)] de la Corte Internacional de Justicia de la Haya. El Tribunal denegó el writ of certiorari de la petición de habeas corpus, entre otras razones, por la emisión de un Memorándum por parte del Presidente de los Estados Unidos expresando la voluntad de dicho país de dar cumplimiento a la citada sentencia de la La Haya, y devolvió el asunto al tribunal juzgador. Con toda probabilidad, este regresará al Tribunal, una vez se produzca la decisión final del tribunal juzgador.

\section{Cláusula de Igualdad}

En Jhonson v. Califorina et al. [03-636], certoriaria del Noveno Circuito, el Tribunal impuso el "escutinio estricto" a la hora de determinar el posible carácter racista de la política (sin base normativa) del Departamento Correccional de Califorina, conforme a la cual los detenidos son segredados racialmente durante los 60 primeros días de su internamiento para evitar "problemas raciales".

\section{Libertad religiosa y Establishment Clause}

En Van Orden v. Perry, in his oficial capacity as Governor of Texas and Chairman, State Preservation Boarda, et al. [03-1500] certiorari del Quinto Circuito, el Tribunal sostuvo que el monolito que se alza junto al Capitolio del Estado de Texas, erigido por la Fraternal Order of Eagles (una organización "Social, civil y patriótica") no viola la laicidad estatal, sino que tan sólo reconoce los méritos de la citada organización.

En McCreary County, Kentucky, et al. v. American Civil Liberties Union of Kentucky et al. [03-1693], certiorari del Sexto Circuito, en cambio, el Tribunal sostuvo la inconstitucionalidad de la exhibición de grandes carteles con los Diez Mandamientos en los juzgados del condado, al considerarlo un mensaje puramente religioso por su falta de integración con otros posibles contenidos laicos.

Sobre garantías penales y procesales

En Illinois v. Caballes [03-923], certiorari procedente del Tribunal Supremo de Illinois, el Tribunal sostuvo que la revisión de un vehículo con perros en- 
trenados para la detección de sustancias psicotrópicas, realizada en un vehículo lícitamente detenido por la policía y que no reveló información distinta de la posesión por parte del conductor del vehículo de sustancias prohibidas, es lícita porque no constituye un "registro" en el sentido de la Cuarta Enmienda.

En United States v. Booker [04-104], certiorari del Séptimo Circuito, el Tribunal ha vuelto a relativizar la eficacia Sentencing Reform Act de 1984 [Pub. L. No. 98-473, tit. II, ch. II, 98 Stat. 1837, 1987] en la línea iniciada en Apprendiv. New Jersey [530 U.S. 466 (2000)] y Blakely v. Washington [124 S. Ct. 2531 (2004)] por su posible conflicto con la Sexta Enmienda.

Derecho electoral

En Clingman, Secretary, Oklahoma State Election Board, et. al. v. Beaver et al. [04-37], certorari del Décimo Circuito, el Tribunal Sostuvo la constitucionalidad de la ley electoral de Oklahoma [OKLA. STAT. ANN. tit. 26, \$1-104(A), (B)(1) (West 1997)] que restringe la posiblidad de participar en elecciones primarias a los afiliados del propio partido y a los independientes, pero excluye a los afiliados de otros partidos.

Propiedad Intelectual

En la larga serie de casos tipo "Napster", el Tribunal en Metro-Goldwyn-Mayer Studios Inc., et al. v. Grokster, Ltd. et al. [04-480], certiorari del Noveno Circuito, ha sostenido que la distribución de dispositivos con el objetivo de promover su uso para infringir los derechos de copyright, tal como se deduce bien de la afirmación directa de tal finalidad o de pasos concretos en dicha dirección, que sobrepase la mera distribución a sabiendas de la acción de terceros, implica responsabilidad ante los actos de violación de los derechos de copyright por terceros, sin perjuicio de los usos lícitos del dispositivo.

\section{Extranjería}

En Clark, Field Office Director, Seattle, Immigration and Customs Enforcement, et al. v. Martinez [03-878], certiorari del Noveno Circuito, el Tribunal ha extendido la regla según la cual el "plazo razonable" de seis meses en que un inmigrante, que ingresó legalmente al país pero del que se ha dispuesto la expulsión, puede permanecer detenido, también para aquellos que ingresan ilegalmente (y cuya expulsión "ordinaria" debe realizarse en un plazo de 90 días). Superado ese plazo, procede su liberación, o antes si transcurridos los 90 días no se prueba la "previsibilidad" de la expatriación en el citado plazo ampliado de 6 meses.

\section{Doctrina Chevron}

Chevron U.S.A. Inc. v. Natural Resources Defense Council, Inc (467 U.S. at 865-66) estableció el principio general de que «las ambigüedad en las leyes dentro de la jurisdicción delegada a una agencia, constituyen delegaciones a dicha 
agencia para colmar estas lagunas de forma razonable ", en National Cable $\varepsilon$ Telecommunications Association et al. v. Brand X Internet Services et al. [04227], certiorari del Noveno Circuito, el Tribunal ha intentado conciliar la tensión entre dicha doctrina en favor de la de la deferencia administrativa y el stare decisis. El Tribunal ha sostenido que una interpretación judicial previa de una ley inválida la de la agencia que, de otro modo, tendría derecho a la deferencia (doctrina Chevron) sólo si la construcción judicial de la norma se deriva de forma no ambigua de los propios términos de la ley.

Sobre la "Cláusula de comercio"

En certiorari al noveno circuito el Tribunal ha sostenido en Gonzales, Attorney General, et al. v. Raich et al. [03-1454] que la Cláusula de Comercio incluye la facultad de prohibir el cultivo local de marihuana, autorizado por la ley estatal californiana para uso medicinal.

ABSTRACT. The doctrinal production of the USA from year 2005 find contradictions between the politic of the Busch Jr's Administration and the constitutional system of rights, but as we will see, it is imposign the frame of reference in which the judicial review of the acts of the political power should be hased in formal judgements of competence. 